\title{
A Case of Pulmonary Artery Hydatid Cyst Observed on Endobronchial Ultrasound
}

\author{
Aysegul Senturk, ${ }^{1,}$ Mukremin Er, ${ }^{1}$ Aysegul Karalezli, ${ }^{1}$ Zeynep Ilerisoy Yakut, ${ }^{2}$ Ayse Nur Soyturk, ${ }^{1}$ \\ Huseyin Cetin, ${ }^{2}$ and Hatice Canan Hasanoglu ${ }^{3}$ \\ ${ }^{1}$ Department of Pulmonary Diseases, Ankara Ataturk Training and Research Hospital, Ankara, Turkey \\ ${ }^{2}$ Department of Radiology, Ankara Ataturk Training and Research Hospital, Ankara, Turkey \\ ${ }^{3}$ Department of Pulmonary Diseases, Yildirim Beyazit University of Medicine, Ankara, Turkey \\ "Corresponding author: Aysegul Senturk, Department of Pulmonary Diseases, Ankara Ataturk Training and Research Hospital, Ankara, Turkey. Tel: +90-3122912525, Fax: \\ +90-3122912527, E-mail: asenturk1967@yahoo.com
}

Received 2013 November 05; Revised 2014 February 17; Accepted 2014 March 19.

\begin{abstract}
Hydatid cyst (HC) is a parasitic disease that may involve many organs, especially the lung and the liver. Pulmonary artery location of the hydatid cyst is extremely rare, but it may cause life-threatening complications. We report a case of a hydatid cyst that completely filled the left main pulmonary artery and its distal part without cardiac involvement. Thoracic computed tomography showed filling defects in the pulmonary arteries. Endobronchial ultrasound was performed for differential diagnosis and it showed a cystic lesion. Hydatid cyst-specific IgE and hem agglutination test results were positive. In the literature, cases like this in which the diagnosis of pulmonary hydatid cyst is made by endobronchial ultrasound are not usually seen. Although many imaging modalities such as plain chest radiography, cross-sectional imaging (MDCT and MRI), echocardiography and conventional pulmonary angiography have been used in the diagnostic approach, we recommend endobronchial ultrasound for the differential diagnosis of cases with cystic formation.
\end{abstract}

Keywords: Hydatid Cyst, Pulmonary Artery, Endobronchial Ultrasound

\section{Background}

Echinococcosis in an important parasitic infection that is still a common health problem in undeveloped and developing countries (1). The liver is the most common site of involvement, followed by the lung. Hydatid disease is often asymptomatic. Symptoms depend on the size and location of the cyst (2). Pulmonary embolism of hydatid cysts is extremely rare (3). The right atrium, right ventricle, and liver have been reported as embolic origins of such cysts (4). Here, we present a case of embolism of a hydatid cyst inside the left main pulmonary artery. The cyst was in the upper segmental branch of the lingular artery, and segmental and sub segmental branches of the lower lobe artery. However, no hydatid cysts were found in other organs. This case is interesting because there are only a few cases in which isolated pulmonary artery hydatid cyst without other organ involvement has been reported in the literature. We believe that this case is the first example of pulmonary artery hydatid cyst diagnosed by EBUS.

\section{Case Presentation}

A 48-year-old woman presented with a one-month history of cough. She had no complaints of sputum, chest pain, or dyspnea, but she had a suspicious history of hemoptysis. She had never smoked. She had a dog in her childhood and she also lived in the rural area. Her physical examination was normal. No abnormal signs were observed on her chest radiograph. Pulmonary function tests and laboratory test results were within normal limits except for eosinophilia and minimally elevated D-dimer in the blood test. D-dimer level of the patient was 600 $\mathrm{ng} / \mathrm{mL}$, while normal limits are 0-500. A negative D-dimer result may exclude pulmonary embolism, but there are many reasons that cause D-dimer elevation such as infection, trauma, deep venous thrombosis, and disseminated intravascular coagulation. In this case, the reason of elevated D-dimer was parasitic infection. The patient was treated with 250-mg fluticasone dry powder twice daily in an inhaler and montelukast once daily under a suspicious diagnosis of allergic asthma; she asked for a control examination 2 weeks later. At the control examination, she still complained of coughing. Thoracic computed tomography (CT) was performed to investigate the etiology of cough and her minimally elevated D-dimer level. Filling defects were observed inside the left main pulmonary artery, upper segmental branch of the lingular artery, and segmental and subsegmental branches of the lower lobe artery on 
her thoracic CT angiogram (Figure 1A-D). This finding at the main and superior branch of the lingular artery showed continuity with an $18 \times 16 \mathrm{~mm}$ nodular opacity considered likely to be a cystic embolism. The right pulmonary artery and its branches were clear. Subcutaneous low-molecularweight heparin anticoagulant therapy was administered on the basis of the suspicious diagnosis of pulmonary thromboembolism. Left and right ventricular parameters on echocardiographic evaluation were normal. Systolic pulmonary artery pressure was $25 \mathrm{~mm} \mathrm{Hg}$. No intracardiac thrombi, or any thrombosis in the deep veins of the lower extremities were observed. Clinical and radiological findings were not completely compatible with thromboemboli. Because pulmonary artery filling defects appeared more well-circumscribed and smooth, and they showed continuity with an $18 \times 16 \mathrm{~mm}$ nodular cystic opacity different from usual features of emboli, the other reasons such as pulmonary artery sarcoma and hydatid cyst causing filling defects in the pulmonary artery on CT were investigated. So for assessment of the other differential diagnoses, the patient underwent endobronchial ultrasound (Olympus EvisExera II CV180). An anechoic cystic area was visualized inside the left pulmonary artery (Figure 2). We performed endobronchial Doppler ultrasound to investigate whether it was a cystic lesion that was causing the filling defects inside the pulmonary artery. Doppler ultrasonography revealed a cystic formation that made the vascular filling defect inside the left pulmonary artery. Thoracic magnetic resonance angiography was performed to investigate the cyst in more detail and it revealed cystic formations in the left pulmonary artery (Figure 3A-D). MRI scans revealed the findings of hydatid cysts. The lesions were hyperintense both on T1-W and T2-W MRI images that showed the liquid within the cyst was rich of protein. On MRI examination, either distal branches of the pulmonary artery were not filled with contrast and showed cystic lesions in them. The nature was the same in the lesions on the proximal branches of the pulmonary artery. Indirect hemagglutination and specific IgE tests were performed to confirm the diagnosis of hydatid cyst; both results were positive. Anticoagulant therapy was stopped, and albendazole treatment was initiated. The patient was scanned for frequent locations of organ involvement of hydatid cysts, but no other cysts were observed. We consulted with cardiovascular surgeons for surgery. The cardiovascular surgeons suggested thoracotomy and embolectomy, but the patient refused the operation. The diagnosis of pulmonary artery hydatid cyst was decided by the patient's history of living in an endemic area, thoracic CT, MRI and EBUS findings and also positive serology tests. Both proximal and distal lesions in the pulmonary arteries had the same nature. So anticoagulant therapy was stopped and albenda- zole treatment was administered to the patient. There was no complaint of coughafter one year follow-up visits. The size of the cyst in the left pulmonary artery on control thoracic CT scans shrunk $7.9 \mathrm{~mm}$ in diameter after one year of albendazole treatment (Figure 4A and B). Albendazole therapy was continued for 2 years.

\section{Discussion}

Hydatidosis is a parasitic infection caused by the larval stage of Echinococcus granulosus. The liver (70\%) and lungs (25\%) are the organs most often affected. Although cardiac involvement is observed in only $0.2-3 \%$ of cases, early diagnosis and treatment are important (5). In primary cardiac hydatidosis, larvae usually reach the myocardium via the coronary circulation, the pulmonary circulation, or a patent foramen ovale. Intestinal lymphatic vessels, thoracic duct, superior and inferior vena cava, and hemorrhoidal and pulmonary veins may be involved (6). A variety of cardiac sequelae including sudden cardiac death, massive pulmonary embolism, and pulmonary hypertension may be encountered clinically. Echocardiography, CT, and magnetic resonance imaging (MRI) can be used to identify cardiac hydatidosis (7). The pulmonary arteries are an exceptionally rare location for hydatid cysts involvement. Hydatid cysts located in the pulmonary arteries are frequently the consequence of embolisms from primary cardiac locations (8). In the present case, a hydatid cyst was located inside the left pulmonary artery and its branches, without any cyst in the heart or other organs. Intra-arterial hydatid cysts grow slowly into the lumen, ultimately occluding it. In the pulmonary arteries, this process may occur over a long period. Because pulmonary arteries have adequate pulmonary perfusion through the bronchial arteries, cases may be initially asymptomatic before the presentation of dyspnea. Symptoms can be observed when the cysts compress a vital structure or interrupt blood circulation, which may also lead to anaphylactic shock (9). The present patient only presented with cough and no dyspnea. In this case, the cough might be the result of compression of the left lower bronchus by the cyst. Hydatid cysts usually have a characteristic appearance on MRI; an oval lesion hypo intense and hyper intense on T1-and T2-weighted images, respectively. In this case, the lesions were hyper intense both on T1-W and T2-W MRI images that shows the liquid within the cyst was rich of protein (10). In the differential diagnosis of pulmonary artery hydatid cyst, pulmonary thromboembolism (fat, amniotic fluid, septic emboli, particulate material) and primary arterial tumors such as sarcoma should be considered since they also make intraluminal defects (11). Radiological findings and the patients' clinical features help to make the dif- 

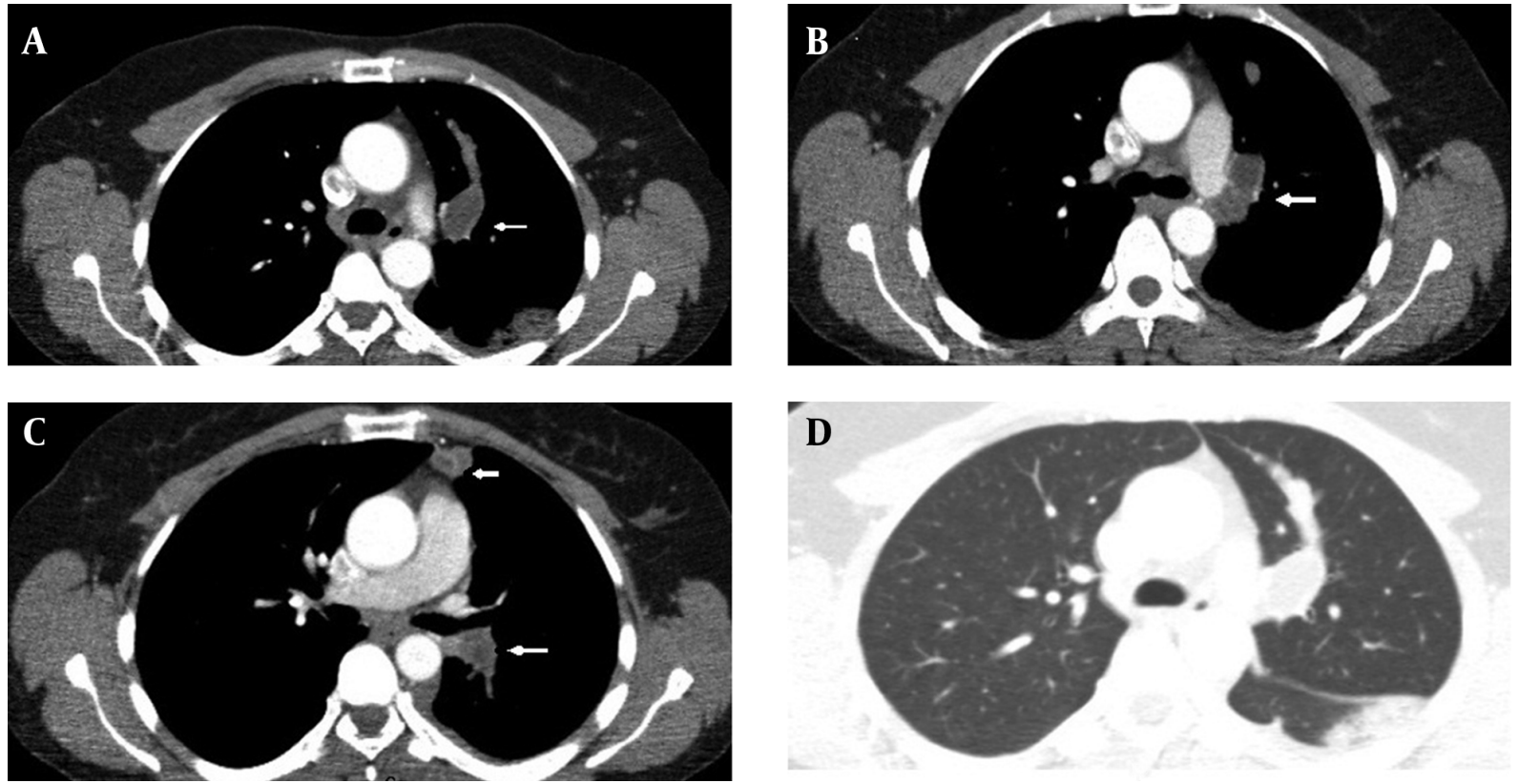

Figure 1. A 48-year-old woman with a one-month history of cough. A-C, CT with contrast shows hypodense masses located in the left main, segmentary branches of the superior lobe and descending pulmonary artery; D, parenchymatous condensation in the superior segment of the lower lobe is seen.

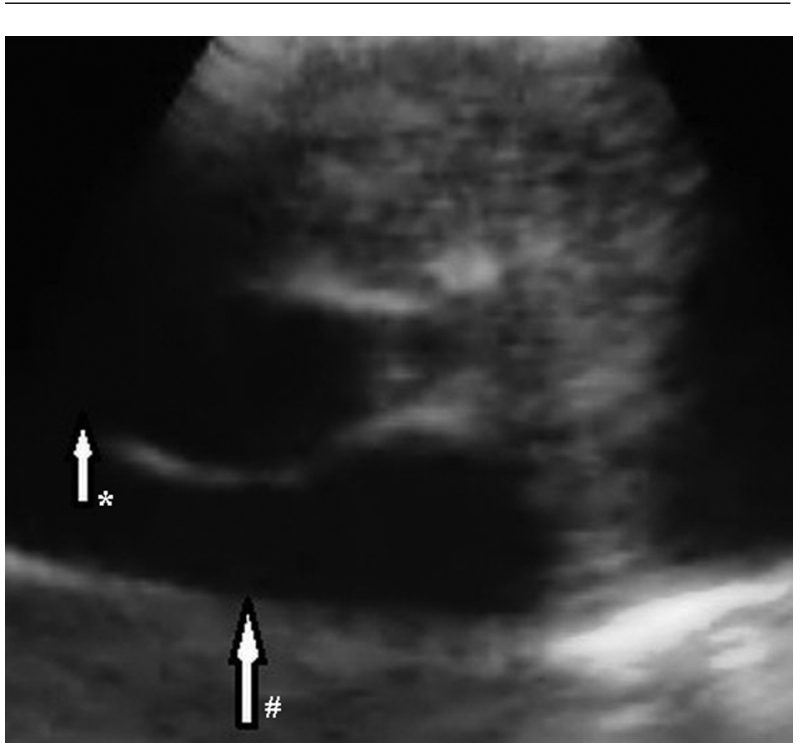

Figure 2. An anechoic cystic lesion is seen inside the left pulmonary artery by EBUS. The cyst in the pulmonary artery is marked with * sign and the pulmonary artery is marked with \# sign.

ferential diagnosis since patients with disseminated pulmonary artery thrombosis show a more aggressive clinical manifestation. Hydatid disease may involve the heart, including the left ventricle (60\%), right ventricle (10\%), peri- cardium (7\%), pulmonary artery (6\%), and interventricular septum (4\%) (12). Because of the significant risk of cyst rupture and anaphylactic shock, surgery should be performed promptly after diagnosis. Extraction of the lesion is recommended, even in asymptomatic patients (13). Echocardiography, spiral CT, MRI, CT angiography, magnetic resonance imaging angiography, and conventional pulmonary angiography can be used for the diagnostic investigation of patients suspected of hydatid pulmonary embolism. If there is no history of hydatid disease, its existence can be suspected based on the presence of anti-echinococcal antibodies and eosinophilia in blood tests (14). The present case had eosinophilia and positive indirect hem agglutination and specific IgE test results. The diagnosis of hydatid cysts mainly depends on radiological and immunological diagnostic tests. Imaging methods are sometimes limited by the small size of the lesion and the atypical images that are difficult to distinguish from abscesses or neoplasms. Routine laboratory diagnosis of hydatid cysts depends on detection of specific antibody response. According to WHO manual for hydatid cysts, CT findings alone allow a correct diagnosis of $61 \%$ hydatid cysts of the liver, lung, kidney, spleen and some other sites and the correct diagnosis rate is $94 \%$ if CT is combined with serology (15). According to a comma, the sensitivity value of ELISA was 92.5\% and the specificity of ELISA was $97.3 \%$. The sensitivity and specificity of IHA was $100 \%$ and $83.3-99 \%$, respectively 

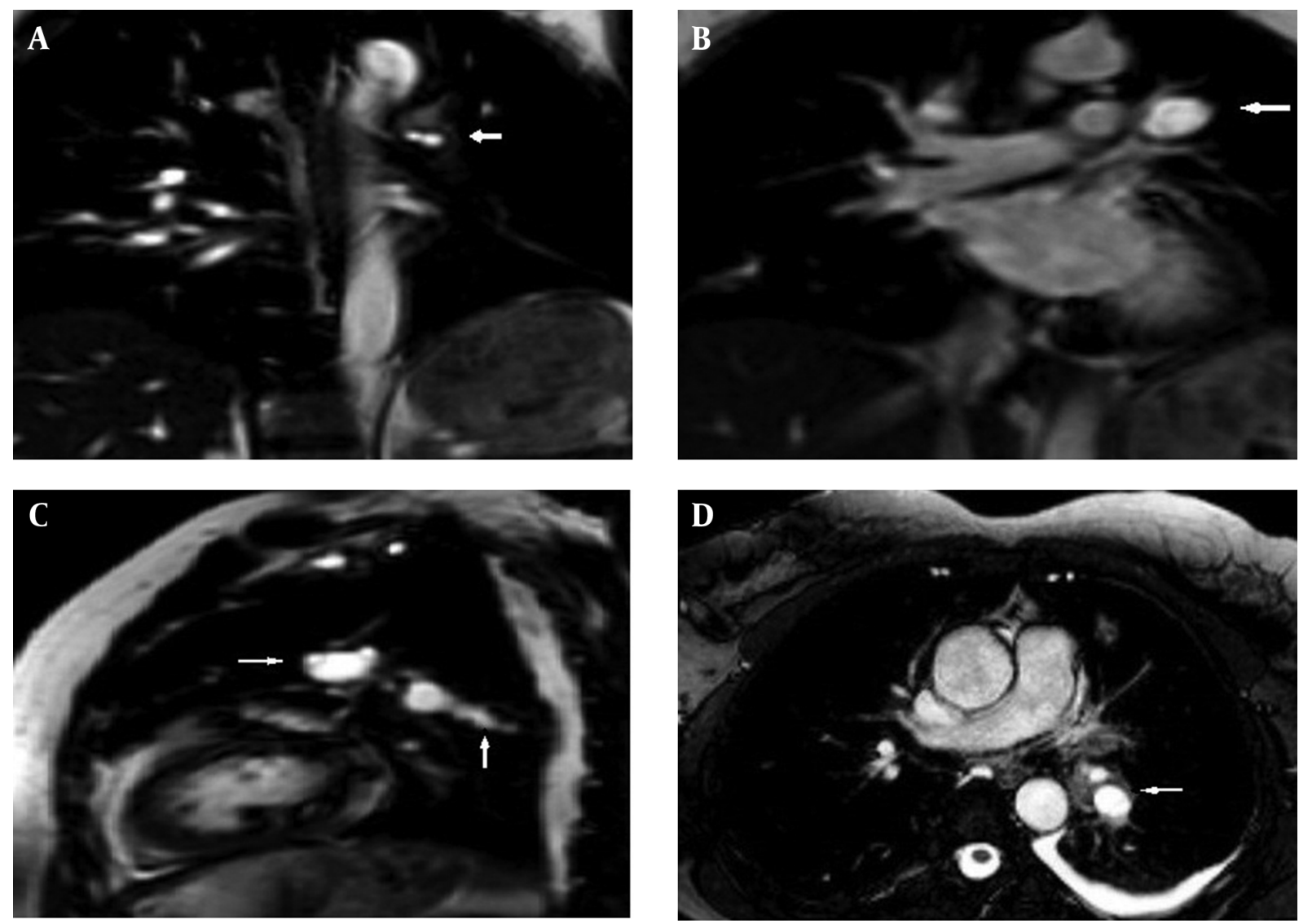

Figure 3. T1 Weighted mri with contrast in coronal; A, B, sagittal; C, and axial D, slices. The main and descending pulmonary arteries not being filled with contrast and hyperintense lesions suggest pulmonary artery hydatid cyst.
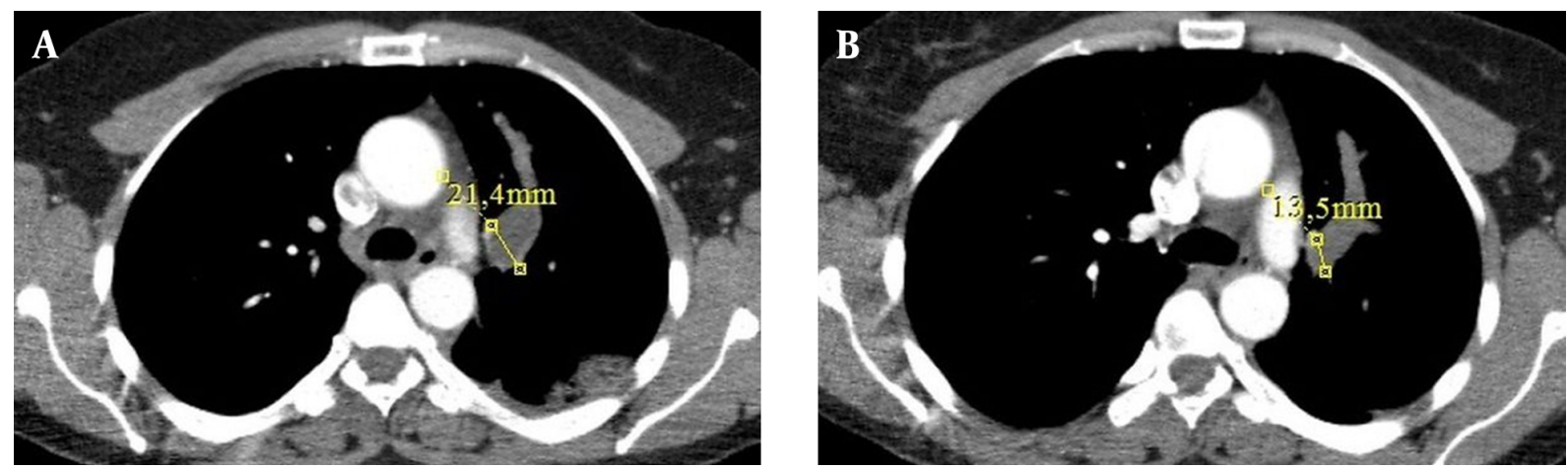

Figure 4. A, B, On control CT scan after one year of therapy, decrease of $7.9 \mathrm{~mm}$ in diameter of the cyst in the left pulmonary artery is seen

(16). However, it should be kept in mind that embolectomy of the hydatid cyst from the main pulmonary truncus increases morbidity and mortality (16). The present patient rejected embolectomy because of its high risks of morbidity and mortality. Many hydatid cysts are asymptomatic and are diagnosed incidentally by clinic or radiology. Most symptomatic cysts have ruptured and may have been secondarily infected (17). Symptoms are usually absent or nonspecific and include chest pain, dyspnea on exercise, and fever. Eosinophilia occurs in $20 \%$ of the cases and may be 
associated with recent rupture (18). Radiological findings range from purely cystic lesions to a completely solid appearance. Hydatid cysts can be solitary or multiple and vary in size (16). In the present case, a cystic filling defect was found inside the main pulmonary artery and a solid defect was found inside the left lower intermediate artery. Rupture into the cardiac chambers occurs most often in the right ventricle and can cause anaphylaxis, or pulmonary or systemic embolism of the daughter cysts (19). Repeated pulmonary emboli may produce pulmonary arterial hypertension and multiple lung cysts (20). In the present case, left ventricular systolic function, the right atrium, and the systolic pulmonary artery pressure were normal and no intracardiac thrombus was found. EBUS is increasingly used for lung cancer staging and the assessment of sarcoidosis (21). It is useful for producing real-time images of both lymph nodes and pulmonary arteries (22). It can be used to diagnose intrapulmonary arterial mass $(22,23)$. After one year of treatment with albendazole, in the follow-up thoracic CT scan, there was a decrease in the size of the cystic lesion in the left pulmonary artery. As a result, albendazole treatment can be an alternative therapy for surgery especially for patients who reject operation.

\section{References}

1. Leila A, Laroussi L, Abdennadher M, Msaad S, Frikha I, Kammoun S. A cardiac hydatid cyst underlying pulmonary embolism: a case report. Pan Afr Med J. 2011;8:12. [PubMed: 22121421].

2. Morar R, Feldman C. Pulmonary echinococcosis. Eur Respir J. 2003;21(6):1069-77. [PubMed: 12797504].

3. Karantanas AH, Bitsios G, Karaiskou E. Echinococcus of the pulmonary artery: CT, MRI and MRA findings. Comput Med Imaging Graph. 2000;24(4):265-7. [PubMed: 10842051].

4. Etievent JP, Vuitton D, Allemand H, Weill F, Gandjbakhch I, Miguet JP. Pulmonary embolism from a parasitic cardiac clot secondary to hepatic alveolar echinococcosis.J Cardiovasc Surg (Torino). 1986;27(6):6714. [PubMed: 3782269].

5. Dighiero J, Canabal EJ, Aguirre CV, Hazan J, Horjales JO. Echinococcus disease of the heart. Circulation. 1958;17(1):127-32. [PubMed:13511630].

6. Kaplan M, Demirtas M, Cimen S, Ozler A. Cardiac hydatid cysts with intracavitary expansion. Ann Thorac Surg. 2001;71(5):1587-90. [PubMed: 11383804]

7. Ege E, Soysal O, Gulculer M, Ozdemir H, Pac M. Cardiac hydatid cyst causing massive pulmonary embolism. Thorac Cardiovasc Surg. 1997;45(5):249-50. doi:10.1055/s-2007-1013738. [PubMed: 9402667].
8. Yague D, Lozano MP, Lample C, Nunez ME, Sanchez F. Bilateral hydatid cyst of pulmonary arteries: MR and CT findings. Eur Radiol. 1998;8(7):1170-2. doi:10.1007/s003300050528. [PubMed: 9724432].

9. Cantoni S, Frola C, Gatto R, Loria F, Terzi MI, Vallebona A. Hydatid cyst of the interventricular septum of the heart: MR findings. AJR Am J Roentgenol. 1993;161(4):753-4. doi: 10.2214/ajr.161.4.8372751. [PubMed: 8372751].

10. von Sinner W, te Strake L, Clark D, Sharif H. MR imaging in hydatid disease. AJR Am J Roentgenol. 1991;157(4):741-5. doi: 10.2214/ajr.157.4.1892028. [PubMed: 1892028].

11. Tardivon AA, Musset D, Maitre S, Brenot F, Dartevelle P, Simonneau $\mathrm{G}$, et al. Role of CT in chronic pulmonary embolism: comparison with pulmonary angiography. J Comput Assist Tomogr. 1993;17(3):34551. [PubMed: 8491891].

12. Dursun M, Terzibasioglu E, Yilmaz R, Cekrezi B, Olgar S, Nisli K, et al. Cardiac hydatid disease: CT and MRI findings. AJR Am J Roentgenol. 2008;190(1):226-32. doi: 10.2214/AJR.07.2035. [PubMed: 18094316].

13. Bakir I, Enc Y, Cicek S. Hydatid Cyst in the Pulmonary Artery: An Uncommon Localization. Heart Surg Forum. 2004;7(1):13-5. [PubMed: 14980840].

14. Bayarogullari H, Davran R, Cavus Y, Yetim TD, Evirgen O. Liver hydatid cyst leading to bilateral pulmonary artery embolism and bilateral multiple pulmonar echinococcosis via inferior vena cava: report of a case. Clin Imaging. 2013;37(2):374-8. doi: 10.1016/j.clinimag.2012.05.008. [PubMed: 23465996].

15. Eckert J, Gemmell MA, Meslin FX, Pawłowski Z. S. . Echinococcosis in humans: clinical aspects, diagnosis and treatment. Paris: WHO;2001.

16. Yapar AF, Aydın M, Kılıc D. , Sükan A, Reyhan M. Intraarterial pulmonary hydatid cysts revealing ventilation-perfusion mismatch on lung scan. Turk J Nucl Med. 2005;14:55-8.

17. Tanju S, Toker A, Ünal ES, Dilege S, Kalaycı G. Hydatid cyst in the distal pulmonary artery: A case report. Turk RespirJ. 2005;6(2):102-4.

18. Radford AJ. In: Oxford textbook of medicine. Weatherall DJ, Ledingham JGG, Warrell DA, editors. Oxford University Press; 1982. pp. 54424.Hydatid disease.

19. Tellez G, Nojek C, Juffe A, Rufilanchas J, O'Connor F, Figuera D. Cardiac echinococcosis: report of 3 cases and review of the literature. Ann Thorac Surg. 1976;21(5):425-30. [PubMed: 131522].

20. Gilsanz V, Campo C, Cue R, Estella J, Estrada RV, Perez-oteiza C, et al. Recurrent pulmonary embolism due to hydatid disease of heart. Study of 3 cases, one with intermittent tricuspid valve obstruction (atrial pseudomyxoma). Br Heart J. 1977;39(5):553-8. [PubMed: 861098].

21. von Bartheld MB, van Breda A, Annema JT. Complication rate of endosonography (endobronchial and endoscopic ultrasound): a systematic review. Respiration. 2014;87(4):343-51. doi:10.1159/000357066. [PubMed: 24434575].

22. Shingyoji M, Ikebe D, Itakura M, Nakajima T, Itami M, Kimura $\mathrm{H}$, et al. Pulmonary artery sarcoma diagnosed by endobronchial ultrasound-guided transbronchial needle aspiration. Ann Thorac Surg. 2013;96(2):e33-5. doi:10.1016/j.athoracsur.2013.01.080. [PubMed: 23910141].

23. Horowitz JC, Kleaveland K, Arenberg D. Endobronchial biopsy of an intrapulmonary arterial mass. J Bronchology Interv Pulmonol. 2013;20(1):93-5. doi: 10.1097/LBR.0b013e3182814b02. [PubMed: 23328154]. 